\title{
The Interaction and Degeneracy of Mixed Solutions for Derivative Nonlinear Schrödinger Equation
}

\author{
Zhen Wu, Shuwei Xu*, Tingwang Wu, Haoqi Zhou \\ College of Mathematics Physics and Information Engineering, Jiaxing University, Jiaxing, China \\ Email: *mailto:xshuwei666@126.com
}

How to cite this paper: $\mathrm{Wu}, \mathrm{Z} ., \mathrm{Xu}, \mathrm{S} . \mathrm{W}$., Wu, T.W. and Zhou, H.Q. (2019) The Interaction and Degeneracy of Mixed Solutions for Derivative Nonlinear Schrödinger Equation. Journal of Applied Mathematics and Physics, 7, 2650-2657.

https://doi.org/10.4236/jamp.2019.711180

Received: October 6, 2019

Accepted: November 2, 2019

Published: November 5, 2019

Copyright $\odot 2019$ by author(s) and Scientific Research Publishing Inc.

This work is licensed under the Creative

Commons Attribution International

License (CC BY 4.0).

http://creativecommons.org/licenses/by/4.0/

\begin{abstract}
The mixed solutions of the derivative nonlinear Schrödinger equation from the trivial seed (zero solution) are derived by using the determinant representation. By adjusting the interaction and degeneracy of mixed solutions, it is possible to obtain different types of solutions: phase solutions, breather solutions, phase-breather solutions and rogue waves.
\end{abstract}

\section{Keywords}

Derivative Nonlinear Schrödinger Equation, Mixed Solutions,

Phase Solutions, Breather Solutions, Rogue Waves

\section{Introduction}

The Derivative Nonlinear Schrödinger(DNLS) equation

$$
i q_{t}-q_{x x}+i\left(q^{2} q^{*}\right)_{x}=0
$$

plays an important role in plasma physics and nonlinear optics. Firstly, the DNLS equation is used to describe the evolution of small but finite amplitude Alfvén waves that propagate quasi-parallel to the magnetic field [1] [2] and large-amplitude magnetohydrodynamic waves in plasmas [3] [4]. Secondly, the DNLS equation governs the propagation of sub-picosecond or femtosecond pulses in single-mode optical fibers [5] [6] [7]. Here “*” denotes the complex conjugation, and subscript of $x$ (or $t$ ) denotes the partial derivative with respect to $x$ (or $t)$.

For the DNLS equation with vanishing boundary condition, Kaup and Newell [8] firstly obtained the one-soliton solutions of the DNLS equation by the inverse scattering transform, and showed that this solution becomes the algebraic 
soliton in a certain limiting condition. Determinant expression of the $\mathrm{N}$-soliton solution [9] for the DNLS equation can be expressed by Darboux transformation. Under non-vanishing boundary conditions, Kawata and Inoue [10] developed an inverse scattering transform of the DNLS equation and introduced the so-called "paired soliton", which is now regarded as the breather solution. With the help of introducing an affine parameter, Chen and Lam [11] revised the inverse scattering transform and then got the single breather solution, which can become the dark soliton and the bright soliton. The rogue waves [12] [13] [14] [15] can be derived from the degeneration of breather solutions by the Darboux transformation [16] [17], which is a very powerful method in integrable nonlinear systems [18] [19] [20] [21].

Rogue waves have recently been studied in a plethora of physical settings, such as deep ocean waves [26], optical fibers [22] [23], and water tanks [24] [25]. The physical mechanisms of rogue wave's generation in many physical systems have been the subject of many research studies [27] [28] [29] [30] [31]. Rogue wave, "appear from nowhere and disappear without a trace" [33], is credited with the Peregrine soliton [32] of the nonlinear Schrödinger (NLS) equation. The Peregrine soliton, which possesses a high amplitude and two hollows, is usually expressed in terms of a simple rational algebraic formula. By the limitation of the infinitely large period of the Kuznetsov-Ma breather [34] [35] and the Akhmediev breather [36] of the NLS equation, the rogue waves can be generated.

The large amplitude waves can be generated from the instability of small amplitude perturbations that are usually chaotic and may contain many frequencies in their spectra. This fact strongly suggests that rogue waves are generally described by adjusting the relative phases of the multiphase solutions and breather solutions of the corresponding nonlinear evolution equations [37] [38] [39].

The aim of this paper is to study the mixed solutions of the DNLS equation and their degeneration mechanism, which implies the obtaining of rogue waves by the synchronization of the mixed solutions: phase solutions and breather solutions. Further, a superposition of mixed solutions may create a hybrid solution, such as a breather solution with periodic conditions, by means of different choices of the phases in the corresponding analytical formulas.

The structure of this paper is as follows. In Section 2, we provide analytically the determinant representation of the mixed solutions. In Section 3, the mixed solutions and their key properties such as the interaction and the degeneration mechanism are discussed. In the limitation $\lambda_{i} \rightarrow \lambda_{c}$, rogue waves, and breather solution with periodic conditions, are generated from the degeneration technique of the mixed solutions: the phase solutions and breather solutions. Finally, we summarize our main results in Section 4.

\section{Mixed Solutions}

The DNLS equation [8] can be given by the integrability condition $U_{t}-V_{x}+[U, V]=0$ of the following Kaup-Newell spectral system (Lax pair) with the reduction condition $r=-q^{*}$. The Lax pairs can be constructed as 
follows:

$$
\begin{gathered}
\partial_{\chi} \psi=\left(J \lambda^{2}+Q \lambda\right) \psi=U \psi, \\
\partial_{t} \psi=\left(2 J \lambda^{4}+V_{3} \lambda^{3}+V_{2} \lambda^{2}+V_{1} \lambda\right) \psi=V \psi,
\end{gathered}
$$

with

$$
\begin{gathered}
\psi=\left(\begin{array}{l}
\phi \\
\varphi
\end{array}\right), \quad J=\left(\begin{array}{cc}
i & 0 \\
0 & -i
\end{array}\right), \quad Q=\left(\begin{array}{ll}
0 & q \\
r & 0
\end{array}\right), \\
V_{3}=2 Q, \quad V_{2}=J q r, \quad V_{1}=\left(\begin{array}{cc}
0 & -i q_{x}+q^{2} r \\
i r_{x}+r^{2} q & 0
\end{array}\right) .
\end{gathered}
$$

here $\lambda$, an arbitrary complex number, is called the eigenvalue (or the spectral parameter), and $\psi$ is called the eigenfunction associated with the eigenvalue $\lambda$ of the Kaup-Newell system.

The general forms of the the mixed solutions [15] are given by using the Darboux transformation and determinant representation of the DNLS equation,

$$
\begin{aligned}
& q^{[2 k+1]}=2 i \frac{\Omega_{11} \Omega_{12}}{\Omega_{11}^{* 2}}, k=1,2, \cdots, \\
& \Omega_{11}=\left|\begin{array}{cccccc}
\lambda_{1}^{2 k} \varphi_{1} & \lambda_{1}^{2 k-1} \phi_{1} & \lambda_{1}^{2 k-2} \varphi_{1} & \cdots & \lambda_{1} \phi_{1} & \varphi_{1} \\
\lambda_{2}^{2 k} \varphi_{2} & \lambda_{2}^{2 k-1} \phi_{2} & \lambda_{2}^{2 k-2} \varphi_{2} & \cdots & \lambda_{2} \phi_{2} & \varphi_{2} \\
\vdots & \vdots & \vdots & \ddots & \vdots & \vdots \\
\lambda_{2 k+1}^{2 k} \varphi_{2 k+1} & \lambda_{2 k+1}^{2 k-1} \phi_{2 k+1} & \lambda_{2 k+1}^{2 k-2} \varphi_{2 k+1} & \cdots & \lambda_{2 k+1} \phi_{2 k+1} & \varphi_{2 k+1}
\end{array}\right|, \\
& \Omega_{12}=\left|\begin{array}{cccccc}
\lambda_{1}^{2 k+1} \phi_{1} & \lambda_{1}^{2 k-1} \phi_{1} & \lambda_{1}^{2 k-2} \varphi_{1} & \cdots & \lambda_{1} \phi_{1} & \varphi_{1} \\
\lambda_{2}^{2 k+1} \phi_{2} & \lambda_{2}^{2 k-1} \phi_{2} & \lambda_{2}^{2 k-2} \varphi_{2} & \cdots & \lambda_{2} \phi_{2} & \varphi_{2} \\
\vdots & \vdots & \vdots & \ddots & \vdots & \vdots \\
\lambda_{2 k+1}^{2 k+1} \phi_{2 k+1} & \lambda_{2 k+1}^{2 k-1} \phi_{2 k+1} & \lambda_{2 k+1}^{2 k-2} \varphi_{2 k+1} & \cdots & \lambda_{2 k+1} \phi_{2 k+1} & \varphi_{2 k+1}
\end{array}\right|, \\
& \phi_{1}=\exp \left[i\left(\lambda_{k}^{2} x+2 \lambda_{k}^{4} t\right)\right], \varphi_{1}=\exp \left[-i\left(\lambda_{k}^{2} x+2 \lambda_{k}^{4} t\right)\right], \lambda_{1}=i h, \\
& \phi_{2 k}=\exp \left[i\left(\lambda_{2 k}^{2} x+2 \lambda_{2 k}^{4} t\right)\right], \varphi_{2 k}=\exp \left[-i\left(\lambda_{2 k}^{2} x+2 \lambda_{2 k}^{4} t\right)\right], \quad \lambda_{2 k}=i l+s_{k} \text {, } \\
& \phi_{2 k+1}=\exp \left[i\left(\lambda_{2 k+1}^{2} x+2 \lambda_{2 k+1}^{4} t\right)\right], \varphi_{2 k+1}=\exp \left[-i\left(\lambda_{2 k+1}^{2} x+2 \lambda_{2 k+1}^{4} t\right)\right], \\
& \lambda_{2 k+1}=i l-s_{k}, s_{k} \in R \bigcup i R \text {. }
\end{aligned}
$$

Notice that the denominators $\Omega_{11}$ are moduli of two non-zero complex functions from Darboux transformation, so the new solution $q^{[2 k+1]}$ is non-singular.

\section{The Interaction and Degeneracy of Mixed Solutions}

The interaction and degeneration of mixed solutions of the DNLS equation can be illustrate by the Equation (6). We mainly consider the function of the parameters $s_{k}$ and $h$ on the generation of the large amplitude waves and rogue waves. For convenience without losing generality, we only consider $k=1$. The density plot of the 2-phase solution $q^{[3]}$ with the fixed parameters $l=\frac{1}{3}$ and 
$h=\frac{1}{3}$ and the variable parameter $s_{1}\left(s_{1}=\frac{1}{5} i, s_{1}=\frac{1}{10} i\right.$, and $\left.s_{1} \rightarrow 0\right)$ is shown in Figure 1. Since the variable parameter $s_{1}$ is a purely imaginary, the phase solutions are given by Equation (6). It can give that the rogue waves can constructed by adjusting the the variable parameter $s_{1}$, i.e. $s_{1} \rightarrow 0$. Given that the variable parameter $s_{1}$ is a real number, the density plot of the 1-breather solution $q^{[3]}$ with the fixed parameters $l=\frac{1}{3}$ and $h=\frac{1}{3}$ and the variable parameter $s_{1}\left(s_{1}=\frac{1}{5}, s_{1}=\frac{1}{10}\right.$, and $\left.s_{1} \rightarrow 0\right)$ is shown in Figure 2. Note that the degeneration procedure of the 2-phase solutions and 1-breather solution is constructed by performing the limitation $s_{1} \rightarrow 0$ and $h=l$, i.e. $\lambda_{k}(k=1,2,3) \rightarrow \lambda_{c}=i l$. In this limitation, we obtain the same rational solution

$$
\begin{gathered}
q_{r}^{[3]}=2 i l \exp \left[-2 i l^{2}\left(-x+2 t l^{2}\right)\right] \frac{\left(H i+i+64 l^{4} t-8 l^{2} x\right)\left(H+8 i l^{2} x-3\right)}{\left(H i+i-64 l^{4} t+8 l^{2} x\right)^{2}}, \\
H=\left(16 l^{4} t-4 l^{2} x\right)^{2}
\end{gathered}
$$

By letting $x \rightarrow \infty, t \rightarrow \infty$, so $\left|q_{r}^{[3]}\right|^{2} \rightarrow 4 l^{2}$, the maximum amplitude of $\left|q_{r}^{[3]}\right|^{2}$ occurs at $(x=0, t=0)$ and is equal to $36 l^{2}$, and the minimum amplitude of $\left|q_{r}^{[3]}\right|^{2}$ occurs at $\left(x=0, t= \pm \frac{\sqrt{3}}{16 l^{4}}\right)$ and is equal to 0 . Obviously, this rational solution is a rogue wave; see the panel (c) in Figure 1 and Figure 2.

In order to have a better understanding of the degeneration procedure of the mixed solutions, a hybrid solution,

$$
\begin{aligned}
q^{[3]}= & -\left(2 \left(16 i \exp \left(-i h^{2}\left(2 h^{2} t-x\right)\right) h^{2} l^{4} t-16 i \exp \left(-i h^{2}\left(2 h^{2} t-x\right)\right) l^{6} t\right.\right. \\
& -4 i \exp \left(-i h^{2}\left(2 h^{2} t-x\right)\right) h^{2} l^{2} x+4 i \exp \left(-i h^{2}\left(2 h^{2} t-x\right)\right) l^{4} x \\
& +\exp \left(-i h^{2}\left(2 h^{2} t-x\right)\right) h^{2}+\exp \left(-i h^{2}\left(2 h^{2} t-x\right)\right) l^{2} \\
& \left.\left.-2 \exp \left(i\left(2 h^{4} t-4 l^{4} t-h^{2} x+2 l^{2} x\right)\right) h l\right)\right)\left(16 i \exp \left(i h^{2}\left(2 h^{2} t-x\right)\right) h^{3} l^{4} t\right. \\
- & 16 i \exp \left(i h^{2}\left(2 h^{2} t-x\right)\right) h l^{6} t-4 i \exp \left(i h^{2}\left(2 h^{2} t-x\right)\right) h^{3} l^{2} x \\
+ & 4 i \exp \left(i h^{2}\left(2 h^{2} t-x\right)\right) h l^{4} x+2 \exp \left(-i\left(2 h^{4} t-4 l^{4} t-h^{2} x+2 l^{2} x\right)\right) l^{3} \\
+ & \left.\exp \left(i h^{2}\left(2 h^{2} t-x\right)\right) h^{3}-3 \exp \left(i h^{2}\left(2 h^{2} t-x\right)\right) h l^{2}\right) \\
& \div\left(16 i \exp \left(i h^{2}\left(2 h^{2} t-x\right)\right) h^{2} l^{4} t-16 i \exp \left(i h^{2}\left(2 h^{2} t-x\right)\right) l^{6} t\right. \\
& -4 i \exp \left(i h^{2}\left(2 h^{2} t-x\right)\right) h^{2} l^{2} x+4 i \exp \left(i h^{2}\left(2 h^{2} t-x\right)\right) l^{4} x \\
& +2 \exp \left(-i\left(2 h^{4} t-4 l^{4} t-h^{2} x+2 l^{2} x\right)\right) h l \\
& \left.-\exp \left(i h^{2}\left(2 h^{2} t-x\right)\right) h^{2}-\exp \left(i h^{2}\left(2 h^{2} t-x\right)\right) l^{2}\right)^{2}
\end{aligned}
$$

is considered as a phase-breather solution shown in Figure 3. 


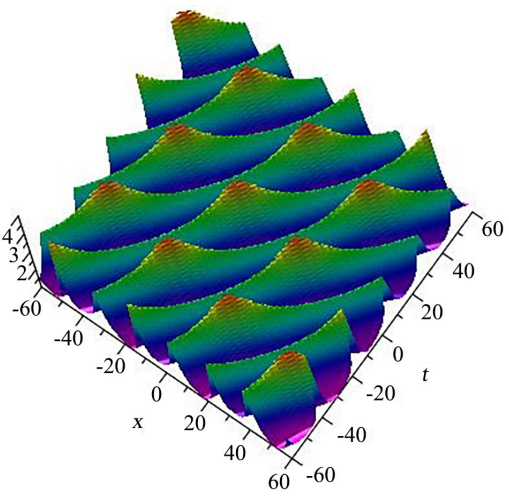

(a)

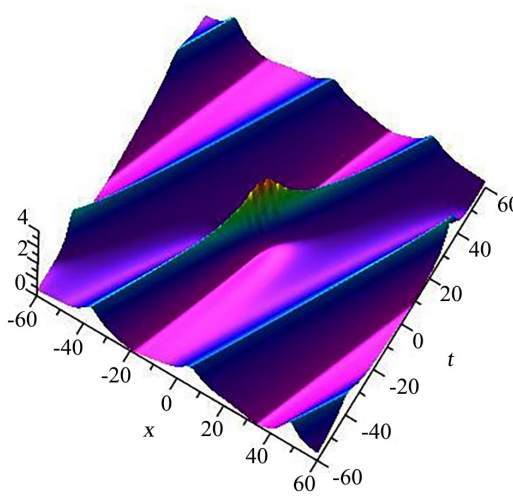

(b)

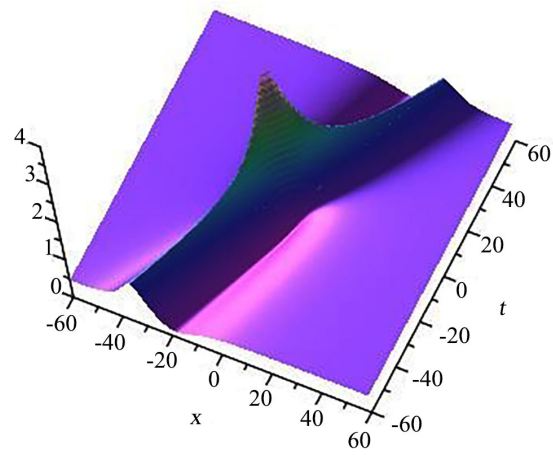

(c)

Figure 1. (Color online) The evolution of profiles for the 2-phase solution $\left|q^{[3]}\right|^{2}$ with the parameters $l=\frac{1}{3}$ and $h=\frac{1}{3}$.

(a) $s_{1}=\frac{1}{5} i$, (b) $s_{1}=\frac{1}{10} i$, (c) $s_{1} \rightarrow 0$.

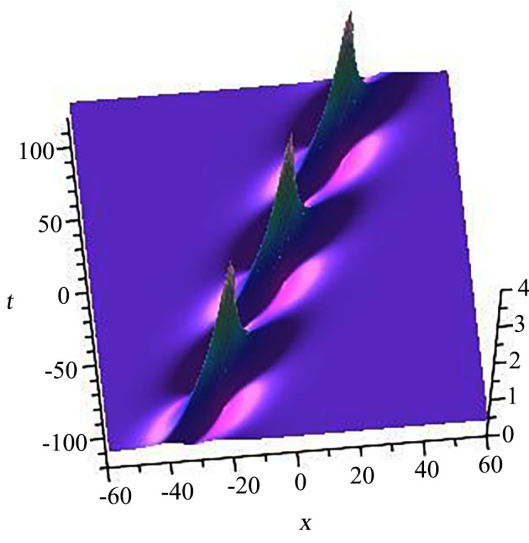

(a)

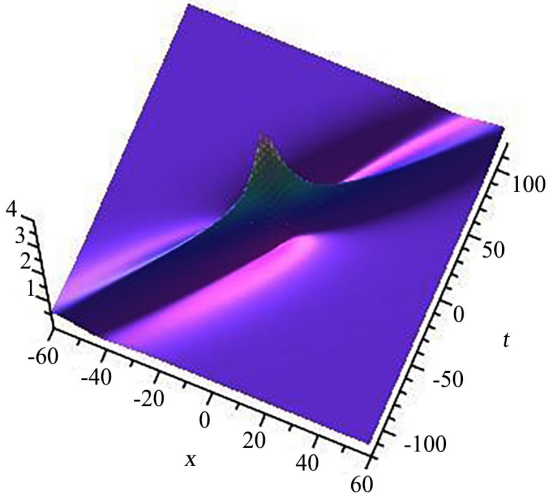

(b)

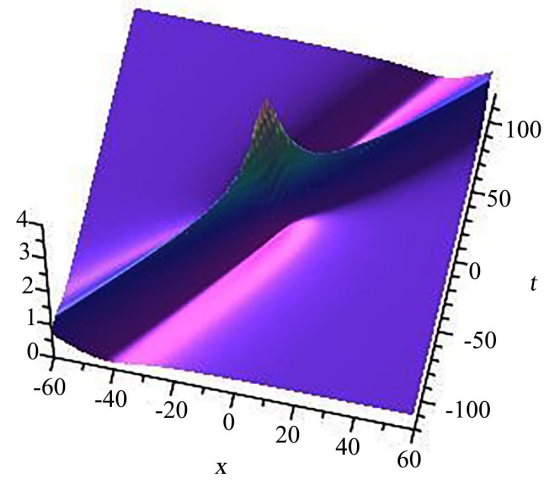

(c)

Figure 2. (Color online) The evolution of profiles for the 1-breather solution $\left|q^{[3]}\right|^{2}$ with the parameters $l=\frac{1}{3}$ and $h=\frac{1}{3}$.

(a) $s_{1}=\frac{1}{5}$, (b) $s_{1}=\frac{1}{10}$, (c) $s_{1} \rightarrow 0$.

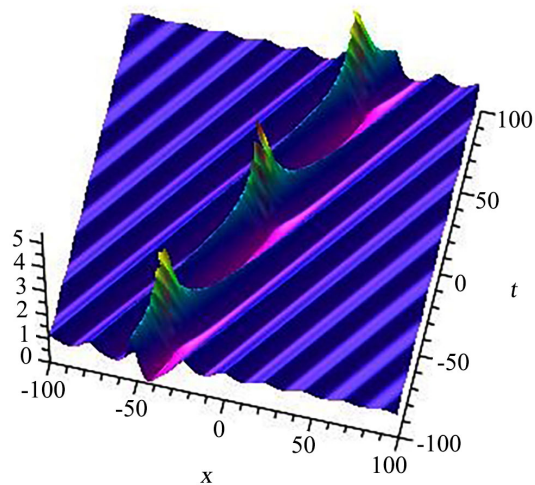

Figure 3. (Color online) The evolution of profiles for the 1-phase-breather solution $\left|q^{[3]}\right|^{2}$ with the parameters $l=\frac{1}{3}, h=\frac{1}{2}$, $s_{1} \rightarrow 0$. 
The solution is constructed by letting $s_{1} \rightarrow 0$, i.e. $\lambda_{2}, \lambda_{3} \rightarrow \lambda_{c}$, where $\lambda_{c}=i l, \lambda_{1}=i h, l=\frac{1}{3}$ and $h=\frac{1}{2}$. Thus by adjusting the relative phases of the mixed solutions in the interacting area, namely, taking the limitation $h \rightarrow l$, i.e. $\lambda_{1} \rightarrow \lambda_{c}$, it can obtain the same rogue wave solution (see in Equation (7)).

\section{Summary}

In this paper, we have shown that rogue waves and some hybrid solutions can be obtained by the synchronization of the mixed solutions: phase solutions and breather solutions. The rogue wave is generated by adjusting the parameters $s_{k}$ and $h$, the limitation $\lambda_{i} \rightarrow \lambda_{c}$, where $\lambda_{c}=i l, l$ being an arbitrary real number. The results provide new insights into the generation of the large amplitude waves and rogue waves, and thus are useful in the application or prevention of the large amplitude waves and rogue waves in the propagation of sub-picosecond or femtosecond pulses. Next we will consider the application of these results to physical theory and experiments and its relation with the initial boundary value problem is also considered.

\section{Acknowledgements}

This work is supported by the National Natural Science Foundation of China under Grant No. 11601187 and Major SRT Project of Jiaxing University.

\section{Conflicts of Interest}

The authors declare no conflicts of interest regarding the publication of this paper.

\section{References}

[1] Mjфlhus, E. (1976) On the Modulational Instability of Hydromagnetic Waves Parallel to the Magnetic Field. Journal of Plasma Physics, 16, 321-334. https://doi.org/10.1017/S0022377800020249

[2] Spangler, S.P. (1997) Nonlinear Waves and Chaos in Space Plasmas. Terrapub, Tokyo, 171.

[3] Ruderman, M.S. (2002) DNLS Equation for Large-Amplitude Solitons Propagating in an Arbitrary Direction in a High- $\beta$ Hall Plasma. Journal of Plasma Physics, 67, 271-276. https://doi.org/10.1017/S002237780200168X

[4] Fedun, V., Ruderman, M.S. and Erdélyi, R. (2008) Generation of Short-Lived LargeAmplitude Magnetohydrodynamic Pulses by Dispersive Focusing. Physics Letters $A$, 372, 6107-6110. https://doi.org/10.1016/j.physleta.2008.08.025

[5] Tzoar, N. and Jain, M. (1981) Self-Phase Modulation in Long-Geometry Optical Waveguide. Physical Review A, 23, 1266-1270. https://doi.org/10.1103/PhysRevA.23.1266

[6] Anderson, D. and Lisak, M. (1983) Nonlinear Asymmetric Self-Phase Modulation and Self-Steepening of Pulses in Long Optical Waveguides. Physical Review A, 27, 1393-1398. https://doi.org/10.1103/PhysRevA.27.1393

[7] Agrawal, G.P. (2001) Nonlinear Fibers Optics. 3rd Edition, Academic, New York. 
[8] Kaup, D.J. and Newell, A.C. (1978) An Exact Solution for a Derivative Nonlinear Schrödinger Equation. Journal of Mathematical Physics, 19, 798-801. https://doi.org/10.1063/1.523737

[9] Huang, N.N. and Chen, Z.Y. (1990) Alfven Solitons. Journal of Physics A: Mathematical and General, 23, 439-453. https://doi.org/10.1088/0305-4470/23/4/014

[10] Kawata, T. and Inoue, H. (1978) Exact Solutions of the Derivative Nonlinear Schrödinger Equation under the Nonvanishing Conditions. Journal of the Physical Society of Japan, 44, 1968-1976. https://doi.org/10.1143/JPSJ.44.1968

[11] Chen, X.J. and Lam, W.K. (2004) Inverse Scattering Transform for the Derivative Nonlinear Schrödinger Equation with Nonvanishing Boundary Conditions. Physical Review E, 69, Article ID: 066604. https://doi.org/10.1103/PhysRevE.69.066604

[12] Xu, S.W., He, J.S. and Wang, L.H. (2011) The Darboux Transformation of the Derivative Nonlinear Schrödinger Equation. Journal of Physics A: Mathematical and Theoretical, 44, Article ID: 305203. https://doi.org/10.1088/1751-8113/44/30/305203

[13] Guo, B.L., Ling, L.M. and Liu, Q.P. (2012) High-Order Solutions and Generalized Darboux Transformations of Derivative Nonlinear Schrödinger Equations. Studies in Applied Mathematics, 130, 317-344. https://doi.org/10.1111/j.1467-9590.2012.00568.x

[14] Xu, S.W., Wang, L.H., Erdlyi, R. and He, J.S. (2019) Degeneracy in Bright-Dark Solitons of the Derivative Nonlinear Schrodinger Equation. Applied Mathematics Letters, 87, 64-72. https://doi.org/10.1016/j.aml.2018.07.021

[15] Xu, S.W., He, J.S. and Mihalache, D. (2019) Rogue Waves Generation through Multiphase Solutions Degeneration for the Derivative Nonlinear Schrodinger Equation. Nonlinear Dynamics, 97, 2443-2452. https://doi.org/10.1007/s11071-019-05140-0

[16] Imai, K. (1999) Generalization of Kaup-Newell Inverse Scattering Formulation and Darboux Transformation. Journal of the Physical Society of Japan, 68, 355-359. https://doi.org/10.1143/JPSJ.68.355

[17] Steudel, H. (2003) The Hierarchy of Multi-Soliton Solutions of the Derivative Nonlinear Schrödinger Equation. Journal of Physics A: Mathematical and General, 36, 1931-1946. https://doi.org/10.1088/0305-4470/36/7/309

[18] Neugebauer, G. and Meinel, R. (1984) General N-Soliton Solution of the AKNS Class on Arbitrary Background. Physics Letters A, 100, 467-470. https://doi.org/10.1016/0375-9601(84)90827-2

[19] Matveev, V.B. and Salle, M.A. (1991) Darboux Transfromations and Solitons. Springer, Berlin. https://doi.org/10.1007/978-3-662-00922-2

[20] Ablowitz, M.J., Kaup, D.J., Newell, A.C. and Segur, H. (1973) Nonlinear Evolution Equations of Physical Significance. Physical Review Letters, 31, 125-127. https://doi.org/10.1103/PhysRevLett.31.125

[21] Ablowitz, M.J. and Clarkson, P.A. (1991) Solitons, Nonlinear Evolution Equations and Inverse Scattering. Cambridge University Press, Cambridge. https://doi.org/10.1017/CBO9780511623998

[22] Solli, D.R., Ropers, C., Koonath, P. and Jalali, B. (2007) Optical Rogue Waves. Nature, 450, 1054-1057. https://doi.org/10.1038/nature06402

[23] Kibler, B., Fatome, J., Finot, C., Millot, G., Dias, F., Genty, G., Akhmediev, N. and Dudley, J.M. (2010) The Peregrine Soliton in Nonlinear Fibre Optics. Nature Physics, 6, 790-795. https://doi.org/10.1038/nphys1740

[24] Chabchoub, A., Hoffmann, N.P. and Akhmediev, N. (2011) Rogue Wave Observa- 
tion in a Water Wave Tank. Physical Review Letters, 106, Article ID: 204502. https://doi.org/10.1103/PhysRevLett.106.204502

[25] Chabchoub, A., Hoffmann, N., Onorato, M. and Akhmediev, N. (2012) Super Rogue Waves: Observation of a Higher-Order Breather in Water Waves. Physical Review X, 2, Article ID: 011015. https://doi.org/10.1103/PhysRevX.2.011015

[26] Kharif, C., Pelinovsky, E. and Slunyaev, A. (2009) Rogue Waves in the Ocean. Springer, Berlin.

[27] Onorato, M., Residori, S., Bortolozzo, U., Montina, A. and Arecchi, F.T. (2013) Rogue Waves and Their Generating Mechanisms in Different Physical Contexts. Physics Reports, 528, 47-89. https://doi.org/10.1016/j.physrep.2013.03.001

[28] Akhmediev, N., Dydley, J.M., Solli, D.R. and Turitsyn, S.K. (2013) Recent Progress in Investigating Optical Rogue Waves. Journal of Optics, 15, Article ID: 060201. https://doi.org/10.1088/2040-8978/15/6/060201

[29] Dudley, J.M., Dias, F., Erkintalo, M. and Genty, G. (2014) Instabilities, Breathers and Rogue Waves in Optics. Nature Photonics, 8, 755-764. https://doi.org/10.1038/nphoton.2014.220

[30] Chen, S., Baronio, F., Soto-Crespo, J.M., Grelu, P. and Mihalache, D. (2017) Versatile Rogue Waves in Scalar, Vector, and Multidimensional Nonlinear Systems. Journal of Physics A: Mathematical and Theoretical, 50, Article ID: 463001. https://doi.org/10.1088/1751-8121/aa8f00

[31] Mihalache, D. (2017) Multidimensional Localized Structures in Optical and Matter-Wave Media: A Topical Survey of Recent Literature. Romanian Reports in Physics, 69, 403.

[32] Peregrine, D.H. (1983) Water Waves, Nonlinear Schrödinger Equations and Their Solutions. The Journal of the Australian Mathematical Society. Series B, Applied Mathematics, 25, 16-43. https://doi.org/10.1017/S0334270000003891

[33] Akhmediev, N., Ankiewicz, A. and Taki, M. (2009) Waves That Appear from Nowhere and Disappear without a Trace. Physics Letters A, 373, 675-678. https://doi.org/10.1016/j.physleta.2008.12.036

[34] Kuznetsov, E.A. (1977) Solitons in a Parametrically Unstable Plasma. Soviet Physics-Doklady, 22, 507-508.

[35] Ma, Y.C. (1979) The Perturbed Plane-Wave Solutions of the Cubic Schrdinger Equation. Studies in Applied Mathematics, 60, 43-58. https://doi.org/10.1002/sapm197960143

[36] Akhmediev, N.N. and Korneev, V.I. (1987) Modulation Instability and Periodic Solutions of Nonlinear Schrödinger Equation. Theoretical and Mathematical Physics, 69, 1089-1093. https://doi.org/10.1007/BF01037866

[37] Bertola, M., El, G.A. and Tovbis, A. (2016) Rogue Waves in Multiphase Solutions of the Focusing Nonlinear Schrödinger Equation. Proceedings of the Royal Society A, 472, Article ID: 20160340. https://doi.org/10.1098/rspa.2016.0340

[38] Slunyaev, A.V. and Pelinovsky, E.N. (2016) Role of Multiple Soliton Interactions in the Generation of Rogue Waves: The Modified Korteweg-de Vries Framework. Physical Review Letters, 117, Article ID: 214501. https://doi.org/10.1103/PhysRevLett.117.214501

[39] Wang, L.H., He, J.S., Xu, H., Wang, J. and Porsezian, K. (2017) Generation of Higher-Order Rogue Waves from Multi-Breathers by Double Degeneracy in an Optical Fiber. Physical Review E, 95, Article ID: 042217. https://doi.org/10.1103/PhysRevE.95.042217 